\title{
Effect of Intravenous Bolus Infusion of Dexmedetomidine on the Duration of Epidural Analgesia with Ropivacaine for Below Umbilical Surgeries in Adult Patients
}

\author{
Rakeshkumar $B^{1}$, Deepak Falgunan ${ }^{2}$ \\ Assistant Professor, Department of Anaesthesia, Mount Zion Medical college, Chayalode, Adoor, Kerala, ${ }^{2}$ Assistant Professor, Department of \\ Anaesthesia, Kerala Medical college, Mangode, Palakkad, Kerala.
}

\section{Abstract}

Background: Epidural anaesthesia is one of the most popular, reliable, safe and easy neuraxial technique to administer and widely used procedure for operative anaesthesia, obstetric analgesia, postoperative pain control and chronic pain management. Epidural anaesthesia is obtained by blocking the spinal nerves in epidural space as the nerves emerge from the dura and pass in to the intervertebral foramina. Duration of epidural anaesthesia may be prolonged by addition of opioids, clonidine, neostigmine, or vasoconstrictor agents to the local anesthetic drug for better post operative pain relief. This study is designed to investigate the effect of intravenous bolus infusion of dexmedetomidine on the duration of epidural analgesia with ropivacaine for below umbilical surgeries in adult patients and its associated adverse events. Aims of study: To determine the effect of intravenous bolus infusion of dexmedetomidine on the duration of epidural analgesia with ropivacaine for below umbilical surgeries in adult patients and to assess the incidence of intra operative side-effects. Subjects and Methods: A double blind prospective randomised control study was done. Patients were allocated in to two study groups, named A and B using computer generated randomization. Results: The duration of analgesia of epidural block with isobaric $0.5 \%$ ropivacaine with intravenous infusion of saline and dexmedetomidine were compared. Post-operative pain was evaluated by Visual Analogue Scale. Duration of analgesia is the time taken from the administration of the drug to the time when the patient complains of pain of $>50$ in Visual Analogue Scale. The duration of analgesia was longest in patients received intravenous dexmedetomidine along with epidural ropivacaine. Conclusion: In conclusion, Intravenous infusion of dexmedetomidine added to epidural block with ropivacaine offered prolonged analgesia in below umbilical Surgeries in adult patients without increasing the incidence of adverse effects.

Keywords: Dexmedetomidine, Isobaric $0.5 \%$ Ropivacaine, Epidural block.

Corresponding Author: Dr. Deepak Falgunan, Assistant Professor, Department of Anaesthesia, Kerala Medical college, Mangode, Palakkad, Kerala.

Received: March 2019

Accepted: March 2019

\section{Introduction}

Central neuraxial blocks with local anaesthetics are popular techniques of anaesthesia which have been extensively used for lower abdominal surgery. Epidural anaesthesia is one of the most popular, reliable, safe and easy neuraxial technique to administer and therefore the widely used procedure for operative anaesthesia, obstetric analgesia, post-operative pain control and chronic pain management. Epidural anaesthesia is obtained by blocking the spinal nerves in epidural space as the nerves emerge from the dura and pass in to the intervertebral foramina.

Ropivacaine has been extensively used since 1996 for regional anaesthesia in adults and older children and has been used safely even in the younger age group for caudal epidural analgesia. ${ }^{[1-4]}$ The lower incidence of cardiovascular side effects and ability to produce less motor blockade has made ropivacaine a safer choice as compared to bupivacaine for epidural anaesthesia especially for day care surgeries. ${ }^{[5]}$
Duration of epidural anaesthesia may be prolonged by addition of opioids, clonidine, neostigmine, or vasoconstrictor agents to the local anesthetic drug for better post operative pain relief. Intrathecal and epidural opioids provide selective analgesia without motor blockade. But they produce significant side effects like delayed respiratory depression, vomiting, pruritus and urinary retention. ${ }^{[6,7]}$ This has prompted further research for non-opiod analgesics with fewer side effects. In this context dexmedetomidine is being extensively evaluated and has been proven to be a potent analgesic.

Dexmedetomidine is a highly selective alpha agonist with potential utility in clinical anaesthesia for its sedative and sympatholytic properties. ${ }^{[2,8]}$ Addition of dexmedetomidine to ropivacaine for epidural analgesia causes prolongation of sensory and motor blockade and duration of post operative analgesia (Paula et al). ${ }^{[9]}$

This study is designed to investigate the effect of intravenous bolus infusion of dexmedetomidine on the duration of 
epidural analgesia with ropivacaine for below umbilical surgeries in adult patients and its associated adverse events.

\section{Aims and Objectives}

To determine the effect of intravenous bolus infusion of dexmedetomidine on the duration of epidural analgesia withropivacaine for below umbilical surgeries in adult patients and to assess the incidence of intra operative sideeffects.

\section{Subjects and Methods}

This study was done in the Department of Anaesthesiology, kerala Medical College Hospital, palakkad, Kerala from June 2016 to December 2016.

\section{Inclusion criteria:}

1. ASA Grade I and II patients.

2. Age Group: $18-55$ years.

3. Body Weight: $60-75 \mathrm{~kg}$.

4. Height: $160-170 \mathrm{~cm}$.

5. Either sex

\section{Exclusion criteria:}

1. Patient refusal for regional anaesthesia.

2. Infection at the site of injection.

3. History of bleeding diathesis and patients on anticoagulant therapy.

4. Patients with cardiovascular disease, renal disease, liver disorders or neurologic disorders.

5. History of allergy to drugs including local anaesthetics.

6. History of spinal deformities and spine surgery .

7. Psychologically ill/ mentally retarded patients.

8. Pregnancy.

\section{Design of Study}

A double blind prospective randomised control study was done. Patients were allocated in to two study groups, named $\mathrm{A}$ and $\mathrm{B}$ using computer generated randomisation.

Group A: Received Epidural Ropivacaine 0.5\% ( isobaric) 10-12 mL till a sensory level of $\mathrm{T} 8$ was attained and intravenous dexmedetomidine $1 \mu \mathrm{g} / \mathrm{kg}$ bolus infusion in 50 $\mathrm{mL}$ over a period of 10 minutes.

Group B: Received Epidural Ropivacaine 0.5\% (isobaric) 10-12 mL till a sensory level of T8 was attained and Normal saline infusion $50 \mathrm{~mL}$ over a period of 10 minutes.

The guide prepared the intravenous bolus drug for both the study groups. The volume of intravenous bolus dose for groups $\mathrm{A}$ and $\mathrm{B}$ was made the same $(50 \mathrm{~mL})$. For loading dose in group A, Dexmedetomidine $1 \mu \mathrm{g} / \mathrm{kg}$ was taken and made to $50 \mathrm{~mL}$ with distilled water and for group B $50 \mathrm{~mL}$ of normal saline was taken. The bolus intravenous dose was given to the patient using an infusion pump over a period of 10 minutes after establishing epidural anaesthesia. The investigator administered the drugs to the patients in each group as per random allocation and direction of the guide. The investigator monitored the patients in both groups for the onset of sensory blockade, duration of analgesia and for any intra operative side effects. The randomization and observations were analysed only at the end of the study.
Written consents were obtained from the patients after informing them about the study. Assessment method used in this study were explained to the patients. Detailed preanaesthetic check up was done. Physical status of the patients were assessed and graded according to ASA guidelines. Age, weight and height of the patients were noted. The basal heart rate and blood pressure were recorded. Laboratory data of the patients were analysed. Patients were advised to fast overnight for 8 hours.

\section{Methodology}

Pre-medication:

Cap. Omeprazole $20 \mathrm{mg}-1$ in the pre operative night and 1 at 6 am on the day of surgery.

Tab. Ondansetron $4 \mathrm{mg}-1$ in the pre operative night and 1 at 6 am on the day of surgery.

Tab. Alprazolam $0.25 \mathrm{mg}-1$ in the pre operative night and 1 at 6 am on the day of surgery.

\section{Preparation:}

The anaesthesia machine was checked. Laryngoscopes with appropriate sized blades, endotracheal tubes, oropharyngeal airways, stylet and working suction apparatus was kept ready. All the necessary drugs were drawn in syringes, labelled and kept ready before the patient was brought to the operation theatre. Intravenous access was established with an $18 \mathrm{G}$ cannula and maintenance fluid with ringer lactate was started. ECG leads and pulse-oximeter probe were attached. Non-invasive blood pressure monitoring was done.

\section{Procedure:}

With the patient in the lateral position, under strict aseptic precautions, epidural space was identified at the level of L3L4 inter-spinous space through midline approach using an 18 G Tuohy epidural needle with loss of resistance technique. An 18G epidural catheter was threaded through the needle and fixed so as $3 \mathrm{~cm}$ of catheter was placed within the epidural space . Epidural test dose was given with $3 \mathrm{cc}$ of $2 \%$ lignocaine with adrenaline ( 1 in 2,00,000) after a negative aspiration for blood and cerebrospinal fluid. After securing the epidural catheter, patient was placed in supine position and test drug was given in graded doses.

After establishing epidural anaesthesia, infusion of dexmedetomidine / saline infusion was started. As per the randomization done, intravenous infusion of a loading dose of $1 \mu \mathrm{g} / \mathrm{kg}$ dexmedetomidine diluted in distilled water to a total volume of $50 \mathrm{~mL}$ or normal saline $50 \mathrm{~mL}$ was given over a period of 10 minutes using an infusion pump.

The procedure was abandoned following failure of epidural block. General anaesthesia was administered in those patients and were excluded from the study.

\section{Monitoring:}

\section{Monitoring include:}

- Continuous electrocardiography,

- Pulse rate and Spo2 using pulse-oximetry and

- Blood pressure using automated non-invasive blood pressure.

Monitoring was recorded at 3 minutes interval for the first 10 minutes and thereafter every 5 minutes till break through pain. 


\section{Assessment:}

- Time of onset of sensory blockade.( Time taken from the administration of epidural block to the attainment of $\mathrm{T} 8$ level.)

- Time to achieve maximum sensory blockade.(Time taken from the administration of epidural block to the attainment of T6 level.)

- Time at which patient complains of pain.

- Duration of analgesia was the time taken from the administration of the drug to the time when the patient complains of pain of $>50$ in visual Analogue Scale.

Onset of sensory block was assessed by pin prick method at every 3 minutes along the mid-clavicular line bilaterally till adequate analgesia (T8 level) was attained.

Duration of analgesia was recorded every 30 minutes during the peri-operative period and then every hour till the occurrence of break through pain.

\section{Duration of analgesia:}

Post-operative analgesia was assessed by Visual Analogue Scale. In this scale ' 0 ' corresponds to no pain and ' 100 ' corresponds to the worst pain possible. Duration of analgesia was the time taken from the time of attainment of epidural anaesthesia to the time when patient complains of pain of $>50$ in the Visual Analogue Scale. Pain was managed with inj.Tramadol and the time was noted. This is the end of study. Adverse effects if any, were noted.

\section{Peri operative side effects:}

The most common Peri operative complications are:

- Hypotension.

- Bradycardia.

- $\quad$ Nausea, Vomiting.

Hypotension is defined as systolic blood pressure $<90 \mathrm{~mm}$ of $\mathrm{Hg}$. Hypotension is managed by injection Mephenteramine $6 \mathrm{mg}$ iv bolus doses.

Bradycardia is defined as heart rate $<50 / \mathrm{mt}$. Bradycardia is managed by injection Atropine $0.6 \mathrm{mg}$ iv bolus doses.

\section{Statistical Analysis}

Data were analyzed using computer software, Statistical Package for Social Sciences (SPSS) version 10. Data are expressed in its frequency and percentage as well as mean, median and standard deviation. To elucidate the associations and comparisons between different parameters, Chi square (c2) test was used as nonparametric test. Student's t test was used as parametric test to compare mean values between two groups. Mann Whitney $U$ test was employed as nonparametric test to compare pain score. For all statistical evaluations, a two-tailed probability of value, $<0.05$ was considered significant. Age group 36-45 years dominated in the dexmedetomidine group and age groups $36-45$ years and 46-55 years dominated in the saline group. Age group 26-35 years represented only $8 \%$ in both groups. As there were no statistical difference $(\mathrm{P}>0.05)$ between the two groups, the distribution of age groups included in this study were comparable. The mean time of onset of sensory blockade in Dexmedetomidine group was 12 minutes and in Saline group was 11.9 minutes. It is not statistically significant with $p$ value $>0.05$. The mean time to achieve maximum sensory blockade was 20.8 minutes in both Dexmedetomidine and
Saline groups.It is not statistically significant with $p$ value $>$ 0.05 .The mean time of first analgesia in Dexmedetomidine group was 368 minutes and in Saline group was 154.4 minutes. It is statistically significant with $p$ value of < 0.05.The mean systolic blood pressure (SBP) in Dexmedetomidine group was $108 \mathrm{~mm}$ of $\mathrm{Hg}$ and in Saline group was $112.6 \mathrm{mmof} \mathrm{Hg}$. It is not statistically significant with $\mathrm{p}$ value $>0.05$. The mean heart rate in Dexmedetomidine group was 70.5 and in Saline group was 74.2. It is not statistically significant with $\mathrm{p}$ value $>0.05$. The distribution of nausea and vomiting in the study population were show with incidence of $4 \%$ in both Dexmedetomidine and saline groups. The study groups were matched as there is no significant difference between the groups.

The pain score was assessed using visual analogue scale at 30 minutes initially then hourly till the pain score reached a score of $>50$. For the first 30 minutes none of the cases in both the groups showed any sign of pain. Whereas the mean pain scores at $60 \mathrm{mts}, 2 \mathrm{hrs}$ and $3 \mathrm{hrs}$ in Dexmedetomidine group was 2.8, 15.6 and 24.4 and in saline group was 19.2, 44 and 50 respectively. Almost all the patients in the saline group showed a pain score of $>50$ before $3 \mathrm{hrs}$ and received rescue analgesia. At $3 \mathrm{hrs}$ the mean pain score in Dexmedetomidine group was only 24.4 . At $60 \mathrm{mts}$, $2 \mathrm{hrs}$, $3 \mathrm{hrs}$, 4hrs, 5hrs $6 \mathrm{hrs}, 7 \mathrm{hrs}$ and $8 \mathrm{hrs}$ the mean pain score in Dexmedetomidine group gradually increased and the values were $2.8, \quad 15.6,24.4,32.1,40,44,47.5$ and 50 respectively.This observed difference among the study groups is statistically significant and dexmedetomidine continues to be a superior drug when mean pain scores were compared between the study groups.

\section{Results}

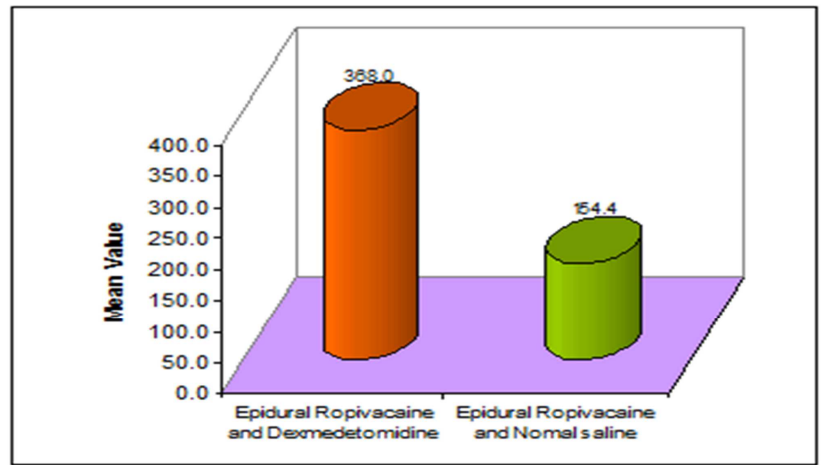

Comparison of time of 1st Analgesia based on group

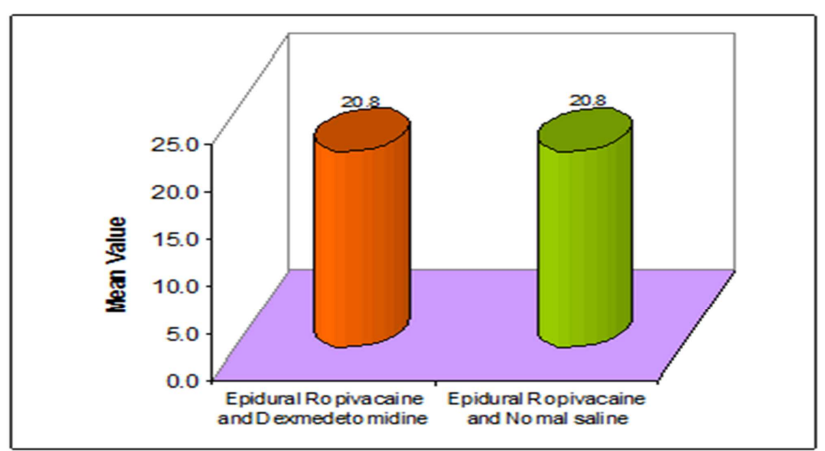

Comparison of time to achieve maximum sensory blockade based on group 


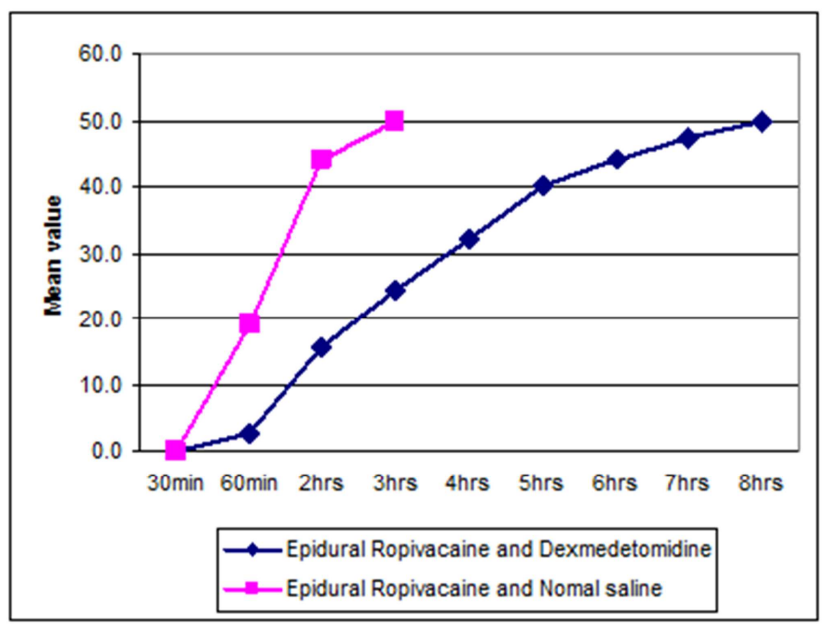

Comparison of pain score based on group at different intervals of time

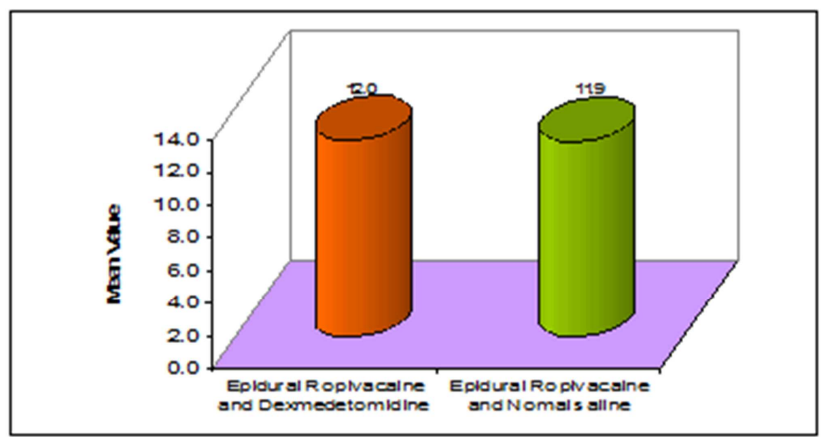

Comparison of time of onset of sensory blockade based on group (Min)

\section{Discussion}

In this study, in lumbar epidural block using ropivacaine, saline and intravenous infusion of dexmedetomidine were compared for providing analgesia. Other than the test drugs, no other analgesics were administered either pre-operatively or intra-operatively to avoid additive effects.

Dexmedetomidine, an $\alpha 2$ agonist, is pharmacologically related to clonidine, has 8 times more affinity for $\alpha 2$ receptors than clonidine. It produces analgesia by binding to adrenoreceptors in the spinal cord. The analgesic effects of a2-adrenergic agonists could be mediated through supraspinal, spinal, and peripheral actions. It produces sedation and anxiolysis by binding to $\alpha 2$ receptors in the locus caeruleus, which diminishes the release of norepinephrine and inhibits sympathetic activity, thus decreasing heart rate and blood pressure.

Dexmedetomidine has an inhibitory effect on the locus caeruleus (A6 group)located at the brain stem. This supraspinal action could explain the prolongation of epidural anesthesia after intravenous administration of dexmedetomidine. The noradrenergic innervation of the spinal cord arises from the noradrenergic nuclei in the brain stem including thelocus ceruleus, the A5, and the A7 noradrenergic nuclei. Neurons in the locus ceruleus are connected to the noradrenergic nuclei in the brain stem.
Axon terminals of the noradrenergic nuclei reach lamina VII and VIII of the ventral horns of the spinal cord. The activity of the noradrenergic neurons is decreased by agonists acting at $\alpha 2$-adrenergic receptors on the locus ceruleus cell bodies. Therefore, inhibition of the locus ceruleus results in disinhibition of the noradrenergic nuclei and exerted descending inhibitory effect on nociception in the spinal cord. Side effects of dexmedetomidine such as hypotension and bradycardia, are dose dependent, Infusion of loading dose over $10 \mathrm{~min}$ and then infusing the maintenance dose decreases the incidence of those side effects.

The addition of dexmedetomidine as an intravenous adjuvant along with local anaesthetic for achieving the same level of anaesthesia but with a prolonged duration of analgesia increases the margin of safety and reduces the incidence of unwanted motor blockade. This study was conducted keeping these facts in mind.

Tarek M Sarhan in 2005 conducted a study in 34 children, aged 2-4 yrs scheduled for elective hypospadias repair. The aim of the study was to detect the effect of prior intravenous administration of dexmedetomidine on the duration of caudal analgesia with ropivacaine in pediatric patients. The study concluded that Intravenous administration of dexmedetomidine $1 \mu \mathrm{g} / \mathrm{kg}$ over 10 minutes prior to caudal analgesia with ropivacaine has an advantage of prolonging lower post operative pain score and increasing the time of first administration of analgesics.

In this study mean pain scores at $60 \mathrm{mts}, 2 \mathrm{hrs}$ and $3 \mathrm{hrs}$ in group A was 2.8, 15.6 and 24.4 and in group B was 19.2, 44 and 50 respectively. Pain score at these interval is clinically and statistically significant with $p$ value $<0.05$.

Mean time of onset of sensory blockade and mean time to achieve maximum sensory blockade in group A was $12 \mathrm{mts}$ and $20.8 \mathrm{mts}$ and in group B was $11.9 \mathrm{mts}$ and $20.8 \mathrm{mts}$ respectively. It is not statistically significant with $\mathrm{p}$ value > 0.05 .

The mean time of first analgesia in group A was 368 minutes and in group B was 154.4 minutes. It is statistically significant with $\mathrm{p}$ value of $<0.05$.

A fall in blood pressure is to be expected in epidural anaesthesia secondary to the sympathectomy caused by local anesthetic action. .In this study mean systolic blood pressure(SBP) in group A is $108 \mathrm{mmHg}$ and for group B it is $112.56 \mathrm{mmHg}$. No further decrease in SBP occured after infusing dexmedetomidine. It is not statistically and clinically significant with $\mathrm{p}$ value $>0.05$.

Mean heart rate in group A is $70.5 / \mathrm{mt}$ and for group B it is $74.2 / \mathrm{mt}$. No further decrease in heart rate occured after infusing dexmedetomidine. It is not statistically and clinically significant with $\mathrm{p}$ value $>0.05$.

Incidence of nausea and vomiting is $4 \%$ in group A as well as in group B. It is not statistically and clinically significant with $\mathrm{P}$ value $>0.05$.

\section{Conclusion}

In conclusion, intravenous infusion of dexmedetomidine added to epidural block with ropivacaine offers prolonged analgesia in adult patients undergoing below umbilical surgeries, without increasing the incidence of adverse effects. 


\section{References}

1. Habre W, Bergesio R, Johnson C, Hackett P, Joyce D, Sims C, et al. Pharmacokinetics of ropivacaine following caudal analgesia in children. Paediatr Anaesth 2000;10:143-7.

2. Yildiz TS, Korkmaz F, Solak M, Toker K. Clonidine addition prolongs the duration of caudal analgesia. Acta Anaesthesiol Scand 2006;50:501-4.

3. Tripi PA, Palmer JS, Thomas S, Elder JS. Clonidine increases duration of bupivacaine caudal analgesia for ureteroneocystostomy: A doubleblind prospective trial. J Urol 2005;174:1081-3.

4. Joshi W, Connelly NR, Freeman K, Reuben SS. Analgesic effect of clonidine added to bupivacaine $0.125 \%$ in paediatric caudal blockade. Paediatr Anaesth 2004;14:483-6.

5. Hansen TG, Ilett KF, Lim SI, Reid C, Hackett LP, Bergesio R, et al.
Pharmacokinetics and clinical efficacy of long-term postoperative epidural ropivacaine infusion in children. Br J Anaesth 2000; 85: 347-53.

6. Chaney M.A. Side effects of intrathecal and epidural Opioids. Canadian Journal of Anesthesia 1995, 42:891-9033.

7. Liu S. S, Hodgson P S, Moore J. M, Trautman W. J. Dose- response effects $\mathrm{f}$ spinal neostigmine added to bupivacaine spinal anesthesia in volunteers. Anesthesiology 1999, 90:710-717.

8. Hall JE, Uhrich TD , Barney JA, Arain SR , Ebert TJ. Sedative , Amnestic and analgesic properties of small dose dexmedetomidine infusions. Anesth Analg 2000; 90:699-705.

9. Paula F Salgado, Paulo JR, Norma SP, Amalia T. Adding dexmedetomidine to ropivacaine $0.75 \%$ for epidural analgesia . Does it improve the quality of the Anesthesia? Anesthesiology 2005; 103:974.

Copyright: ( ) the author(s), publisher. Academia Anesthesiologica International is an Official Publication of "Society for Health Care \& Research Development". It is an open-access article distributed under the terms of the Creative Commons Attribution Non-Commercial License, which permits unrestricted non-commercial use, distribution, and reproduction in any medium, provided the original work is properly cited.

How to cite this article: Rakeshkumar B, Falgunan D. Effect of Intravenous Bolus Infusion of Dexmedetomidine on the Duration of Epidural Analgesia with Ropivacaine for Below Umbilical Surgeries in Adult Patients. Acad. Anesthesiol. Int. 2019;4(1):19-23.

DOI: dx.doi.org/10.21276/aan.2019.4.1.5

Source of Support: Nil, Conflict of Interest: None declared. 